\title{
CORRECTION OF THE BETATRON COUPLING AND CLOSED ORBIT DISTORTION AT THE VEPP-2000 COLLIDER
}

\author{
I.A. Koop, E.A. Perevedentsev, D.B. Shwartz, A.A. Valishev*, \\ Budker Institute of Nuclear Physics, 630090, Novosibirsk, Russia
}

\begin{abstract}
An essential condition for the round beam mode operation at the VEPP-2000 storage ring is the zero betatron coupling in the arc between two Interaction Points. To comply with this requirement the closed orbit should be corrected and the proper skew quadrupole correction system must exist. This work presents the development of the dipole and skew quadrupole correction systems for VEPP-2000. The calculations of correction of the linear betatron coupling due to solenoids are compared to the measurement results from the VEPP-2M storage ring.
\end{abstract}

\section{INTRODUCTION}

The new electron-positron collider VEPP-2000 with energy up to $2 \times 1 \mathrm{GeV}$ [1] will use the round colliding beams concept [2]. The final focusing will be performed by $13 T$ superconducting solenoids, rotating the betatron oscillations plane by $\pi / 2$. This is the first machine with this type of optics therefore an investigation of the betatron coupling at VEPP-2000 was the main aim of this work. Besides the skew-quadrupole correctors system development a closed orbit correction elements were calculated and designed. Despite of the non-trivial eigenmode behavior in the round beams machine the coupling control turned out to be equivalent to the case of ordinary flat beams. The skewquadrupoles are absolutely necessary for the VEPP-2000 optics, since the betatron mode coupling cannot be controlled by the longitudinal field adjustment due to the absence of betatron phases difference between the solenoids.

\section{CLOSED ORBIT CORRECTION}

The VEPP-2000 ring consists of two identical arcs and two experimental drifts. All correction system elements are designed as additional coils in main elements because of very compact arrangement of optics elements. Thus, the dipole coils are settled on the iron yoke of the quadrupole lenses, skew-quadrupole coils are situated on two poles of the sextupole lenses. All F-lenses and bending magnets contain horizontal dipole correctors, D-lenses contain vertical correctors (Fig. 1). The F1 lens is equiped with both direction correctors to control closed orbit distortions, caused by solenoid displacement. The solenoid and the F2lens shift of $X=0.2 \mathrm{~mm}$ causes $5 \mathrm{~mm}$ closed orbit distortion and requires dipole corrector fields $H=0.015 T$ to

*valishev@inp.nsk.su compensate it. The local closed orbit bump with corrector threes was envisaged and calculated.

\section{BETATRON COUPLING}

In traditional "flat" optics, i.e. in a ring with vertical bending magnet field and without longitudinal magnetic fields the closed orbit lies in horizontal plane, radial betatron oscillations are exited by the synchrotron radiation quantum fluctuations and vertical oscillations appear only due to betatron coupling. To increase the luminosity the vertical emittance must be minimized by coupling suppression. It makes the oscillations independent in two different dimensions. In the ideal lattice the $4 \times 4$ betatron transport matrix from one azimuth to another $T\left(s_{1} \rightarrow s_{2}\right)$ including one turn matrix $M(s)=T(s \rightarrow s)$ is block-diagonal at any azimuth. The two eigenmodes lie in vertical and horizontal planes accordingly. Change in one of the betatron tunes does not affect the another one.

The effect of the element with longitudinal or skewquadrupole field located at some azimuth can not be completely reduced by another elements placed at another azimuth, but for example the one turn matrix $M\left(s_{0}\right)$ can be diagonalized. At the other azimuth $s \neq s_{0}$ the matrix still may be not block-diagonal. The conventional way to compensate the coupling consists in suppression of coupling resonances. Then the so-called "zero coupling" case is the independent betatron tunes control.

The second effect of betatron coupling is excitation of the vertical dispersion function $\eta_{y}$ which leads to vertical emittance growth and also to additional undesirable coupling of betatron and synchrotron oscillations.

In the round beam machine the final focusing solenoids rotate betatron oscillations plane by $\pi / 2$. This case seems to be the case of "absolute" coupling but in fact it is not. Using the parametrization [4] one can see the eigenmodes structure (Fig.2). Vertical oscillation in one arc turns to horizontal in another arc. Modes are excited by the synchrotron radiation fluctuations in different arcs and have equal emittances. In ideal VEPP-2000 lattice there is no coupling between modes.

The solenoid transport matrix contains coupling terms but one turn matrix is block-diagonal at any azimuth. The betatron tunes fractional parts are equal. This corresponds to exact difference resonance whereas coupled oscillations can not have equal tunes the minimal distance exists one can bring them together. Thus the coupling compensation scheme in the round beam machine must be the same as in the flat one. The difference is the equality of horizontal and 


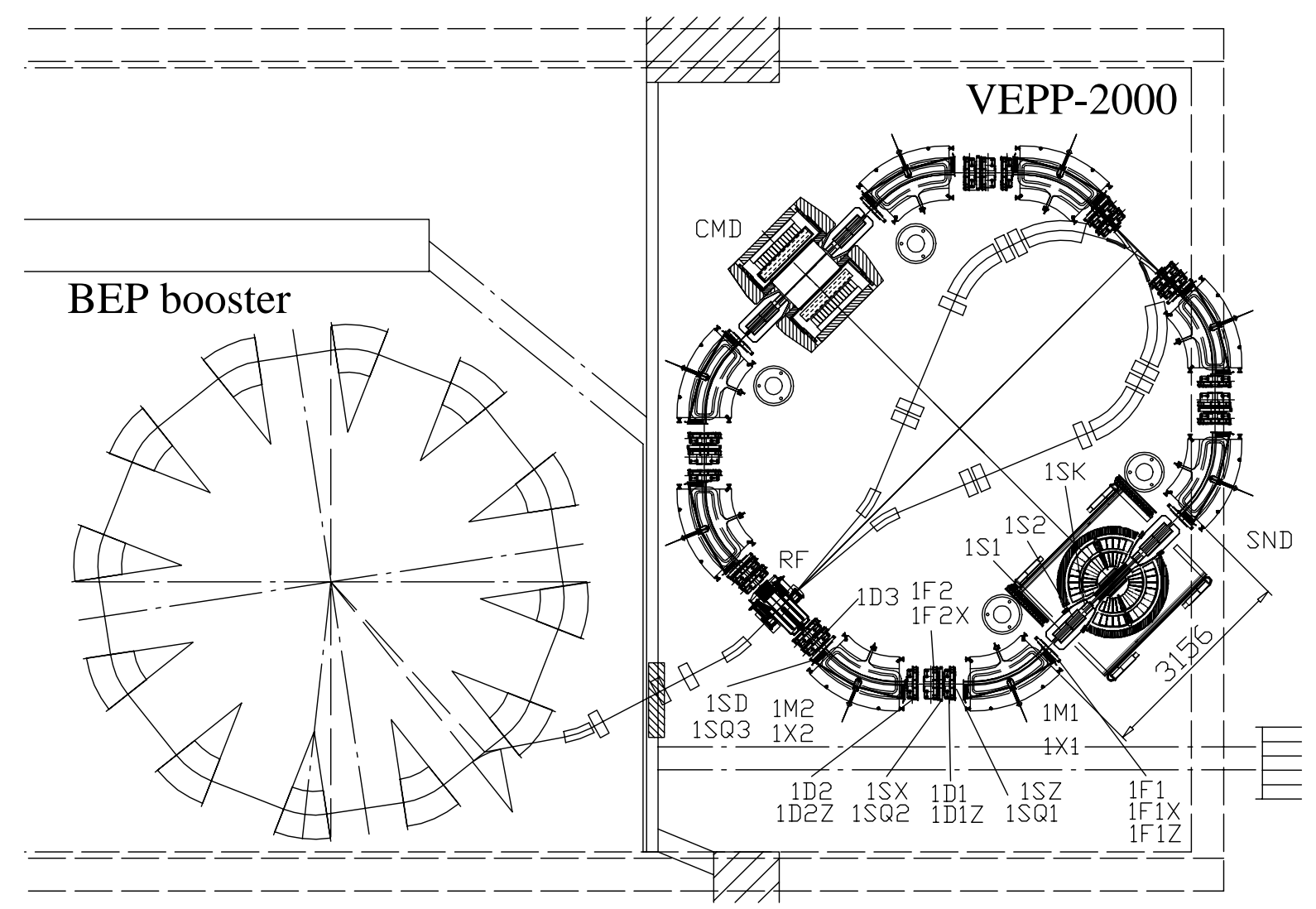

Figure 1: VEPP-2000 collider layout.

vertical emittances but their exact equality does not indicate the absence of mode coupling in contrast with the flat case, where $\varepsilon_{y}=0$ means zero betatron coupling.

From here on the main results come from simulation of a flat lattice with coupling elements. In the presence of two skew-quadrupoles their compensation is possible. In the case of weak coupling near the difference resonanse the tune split is characterized by the integral parameter [5] $\Delta \nu=|C|$,

$$
\begin{gathered}
C=\frac{1}{2 \pi} \oint \sqrt{\beta_{x} \beta_{y}}\left[q+\frac{L}{2}\left(\frac{\alpha_{x}}{\beta_{x}}-\frac{\alpha_{y}}{\beta_{y}}\right)+\imath \frac{L}{2}\left(\frac{1}{\beta_{x}}+\frac{1}{\beta_{y}}\right)\right] \\
\times \exp \left(-\imath\left(\Psi_{x}-\Psi_{y}\right)\right) d s .
\end{gathered}
$$

Here $\beta_{x}, \beta_{y}, \alpha_{x}$ and $\alpha_{y}$ are the Twiss parameters, $q=$ $\left(\partial H_{x} / \partial x\right) / H R$ is the skew quadrupole gradient, $L$ is the longitudinal field $H_{s} / H R, \Psi_{x}$ and $\Psi_{y}$ are the betatron phases. This formula was derived from equations of motion after fast oscillations averaging [6]. It is necessary to minimize both real and imaginary parts of this complex parameter $\mathrm{C}$ to make tunes independent. In the case of two thin skew-quadrupoles the tune split depends only on the difference of betatron phases advance between them $\left(\Delta \Psi_{x}-\right.$ $\left.\Delta \Psi_{y}\right)$. The compensation possible if $\left(\Delta \Psi_{x}-\Delta \Psi_{y}\right)$ is mul- tiple to $\pi$. Effect of a thin skew-quadrupole can be theoretically compensated by one solenoid only in the case of special conditions on lattice functions. Tune split calculations were done using matrix formalism without assumptions about weakness of coupling. For VEPP-2000 lattice it was checked that two skew-quadrupoles located either in one arc or in different arcs make no effect on the tune split assuming that phases advance difference is equal. Simulation of the lattice with one thin skew-quadrupole confirmed the impossibility to compensate its effect with a solenoid. The skew-quadrupole gradient $G=0.5 \mathrm{~T} / \mathrm{m}$ allows to compensate tune split $\Delta n u=0.001$ which is a scale of synchrotron tune. Simulations show that tune split of this magnitude leads to a significant luminosity reduction due to beam-beam effects. It is not possible to make correctors with large reserve in strength because of limited space allowance for the coils.

One of the skew-quadrupole field sources is the sextupole if the closed orbit is shifted from its center. The gradient of VEPP-2000 sextupoles SZ is $2 T / m$. Since the correctors strength is not enough to compensate this coupling source at high energy, it is desirable to limit the closed orbit distortions in sextupoles. 


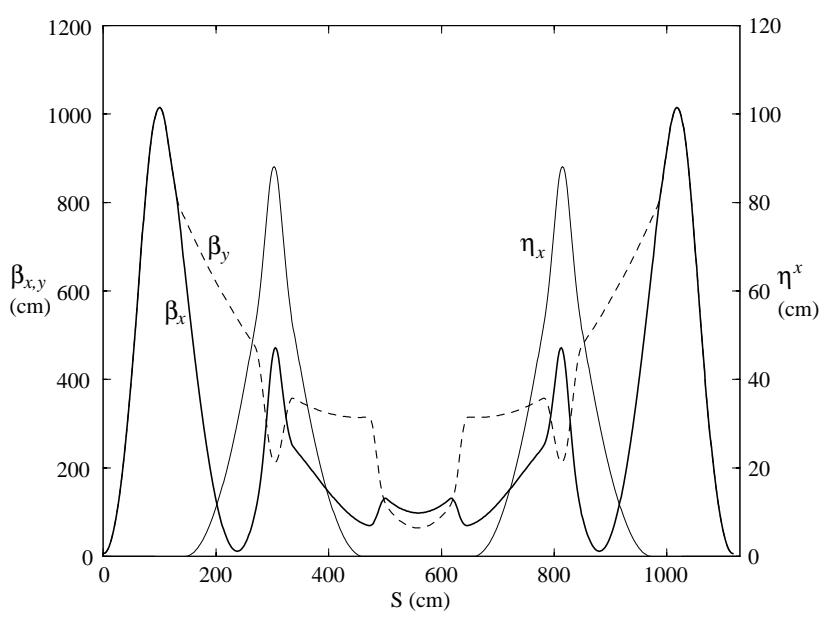

Figure 2: Lattice functions (half period).

\section{EXPERIMENTS AT VEPP-2M}

To check the above theory a test measurements of the weak coupling effects were done at the VEPP-2M storage ring (Novosibirsk, [3]) during the last days of its operation in the year 2000. The ring had two skew-quadrupole families: VSQ (1-8), located in Y-sextupoles and four separate skews GSQ. For the experiment a special solenoid was wound over the vacuum chamber in the technical straight section. It had length of $10 \mathrm{~cm}$ and was capable of creating a longitudinal magnetic field of $0.08 \mathrm{~T}$ at the beam orbit.

During the measurement the $\beta$-functions in the periodicity elements were kept identical with the accuracy of 10$15 \%$ and the betatron tunes were equal. Next, the coupling was eliminated and the tune split became zero. Then some elements (solenoid, skew) were turned on in different combinations and the tunes splitted. The spectrum of coherent betatron oscillations was measured with the scanning spectrum analyzer which took the signal from the pickup electrodes.

The linear dependence of the tune split on the correctors strength was checked, as well as its independence on the working point. A special measurements were done to check a possibility to compensate the effect of the solenoid with two skews.

\section{CONCLUSION}

The closed orbit correction system for the new collider VEPP-2000 with round colliding beams was calculated and designed. Despite of the unusual optics with equal betatron emittances and tunes the necessity of traditional skewquadrupoles system was shown, since the betatron coupling can not be fully controlled by adjustment of the solenoidal field. Skew-quadrupole correctors were calculated and designed.

\section{ACKNOWLEDGMENTS}

The authors thank Yu.M. Shatunov for his interest and support of the work.

\section{REFERENCES}

[1] Yu.M. Shatunov, A.V. Evstigneev, D.I. Ganyushin et al., "Project of a new electron-positron collider VEPP-2000", in Proc. of the 2000 European Particle Acc. Conf., Vienna (2000), p. 439

[2] V.V. Danilov et al., "The concept of round colliding beams", in Proc. of the 1996 European Particle Acc. Conf., Barcelona (1996), p.1149

[3] P.M. Ivanov, I.A. Koop, E.A. Perevedentsev, Yu.M. Shatunov and I.B. Vasserman, "Luminosity and the beam-beam effects in the electron-positron storage ring VEPP-2M with superconducting wiggler magnet", in Proc. 3rd Advanced ICFA Beam Dyn. Workshop, May 29 - June 3, Novosibirsk, 1989, p. 26 (1989).

[4] V.N. Litvinenko and E.A. Perevedentsev, "Evaluation of the Beam Parameters in Storage Rings with Full Linear Coupling", in: Proc. 6th All-Union Conf. on Charged Particles Accelerators, Dubna, 1979, vol. 2, pp. 285-288

[5] E.A. Perevedentsev, "Measurement of Coupling", lecture in: Proc. Joint School on Accel., Montreux, May 1998, World Scientific, 1999

[6] A.A. Kolomensky and A.N. Lebedev, "Theory of Cyclic Accelerators", North-Holland, 1966 